\title{
IMPLEMENTACIÓN DE RECOMENDACIONES DE INFORMES DE AUDITORÍA Y SU INCIDENCIA EN LA GESTIÓN DEL GOBIERNO REGIONAL PUNO
}

\author{
IMPLEMENTATION OF RECOMMENDATIONS OF AUDIT REPORTS AND THEIR INCIDENCE IN \\ THE MANAGEMENT OF THE PUNO REGIONAL GOVERNMENT
}

DOI: https://doi.org/10.47190/rcsaw.v2i3.2

Recibido: 03/01/22

Aceptado: 01/02/22

Ronny Alexander Gutiérrez Castillo

https://orcid.org/0000-0003-0861-8027

alxandrgutcas@gmail.com, Universidad Nacional del Altiplano Puno

\section{RESUMEN}

La implementación de las recomendaciones de los informes de auditoría realizadas al Gobierno Regional de Puno, es un tema importante toda vez que estos deberían incidir positivamente en la gestión de dicha entidad, por ese motivo se planteó como objetivo: Determinar el nivel de cumplimiento en la implementación de las recomendaciones planteadas en los informes de auditoría emitidos por el Órgano de Control Institucional y su incidencia en la gestión del Gobierno Regional de Puno; 2013-2014. La metodología de la investigación posee un enfoque cuantitativo de diseño no experimental de tipo transversal con un nivel descriptivo y analítico; la población estuvo conformada por 79 recomendaciones de las que se realizó un muestro no probabilístico - muestra censal, aplicando el criterio de exclusión se tuvo una muestra de 57 recomendaciones. Se utilizó como técnica de recolección de datos, el análisis documental. Los resultados de la investigación se presentaron en tablas y figuras estadísticas, de las cuales se obtuvo que de las 47 recomendaciones evaluadas solo uno (1) que equivale al $2.13 \%$ fue implementada, 15 que equivale al $31.91 \%$ se encuentran en proceso de implementación y 31 que equivale al $65.96 \%$ están pendientes de implementar. La conclusión a la que se llegó fue que el incumplimiento de la implementación de las recomendaciones planteadas en los informes de auditoría, dan como producto que se tenga una incidencia negativa respecto a la gestión del Gobierno Regional Puno; siendo algunas de las causas la carencia de algún dispositivo legal interno de la entidad, llámese directiva interna que vele por el adecuado cumplimiento en la implementación de las recomendaciones planteadas en los informes de auditoría por parte de las diferentes gerencias y oficinas de la entidad.

Palabras Clave: Control, cumplimiento, gobierno regional e institucional.

\begin{abstract}
The implementation of the recommendations of the audit reports made to the Regional Government of Puno, is an important issue since these should have a positive impact on the management of said entity, for that reason it was proposed as an objective: To determine the level of compliance in the implementation of the recommendations set forth in the audit reports issued by the Institutional Control Body and their impact on the management of the Regional Government of Puno; 2013-2014. The methodology to a research with a quantitative approach of nonexperimental design of transversal type with a descriptive and analytical level; The population was made up of 79 recommendations of those making a non-probabilistic sampling - census sampling, applying exclusion criteria, a sample of 57 recommendations was taken. Documentary analysis was used as a data collection technique. The results of the research were presented in tables and statistical figures, from which it was obtained that of the 47 recommendations evaluated only 1 that is equivalent to $2.13 \%$ was implemented, 15 that is equivalent to $31.91 \%$ are in the process of implementation and 31 that is equivalent $65.96 \%$ are pending implementation. The conclusion reached was that failure to comply with the recommendations set forth in the audit reports, results in a negative impact on the management of the Puno Regional Government; being some of the causes the lack of some internal legal device of the entity, call itself an internal directive that ensures adequate compliance in the implementation of the recommendations raised in the audit reports by the different management and offices of the entity.
\end{abstract}

Keywords: Control, compliance, regional government and institutional.

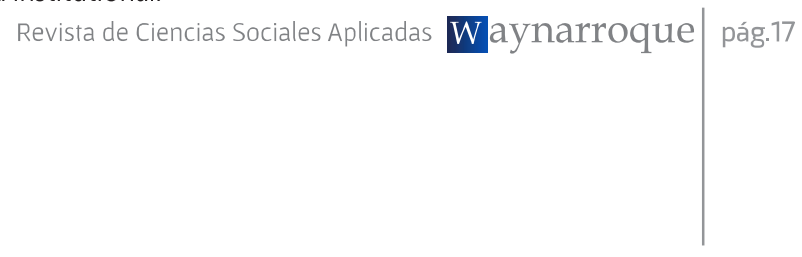




\section{INTRODUCCIÓN}

\section{Sistema Nacional de Control}

La Contraloría General de la República (2002) define al Sistema Nacional de Control como el conjunto de Órganos de Control, normas, métodos y procedimientos (estructurados e integrados funcionalmente) destinados a conducir y desarrollar el ejercicio del control gubernamental en forma descentralizada. Tiene como órgano rector a la Contraloría General de la Republica.

La Contraloría General de la República como ente rector del Sistema Nacional de Control es el encargado de controlar una correcta, eficiente y transparente utilización y gestión de los recursos y bienes pertenecientes al Estado, para lo cual establece mecanismos y políticas.

\section{Órganos del Sistema Nacional de Control}

Conforme a la Ley N.²7785, Ley Orgánica del Sistema Nacional de Control y de la Contraloría General de la República, establece que el sistema está conformado por la Contraloría General (como ente rector), los órganos de auditoría interna y las sociedades de auditoría.

\section{Órgano de Control Institucional}

Según el artículo N. 17 de la Ley n. 27785 Ley Orgánica del Sistema Nacional de Control y de la Contraloría General de la Republica; está ubicado en el mayor nivel jerárquico de la estructura de la entidad, en la cual constituye la unidad especializada responsable de llevar a cabo el control gubernamental en la entidad. Teniendo entre sus funciones: "Efectuar el seguimiento de las medidas correctivas que implemente la entidad como resultado de las labores de control, comprobando y calificando su materialización efectiva, conforme a las disposiciones de la materia".

\section{Auditoria Gubernamental}

La auditoría es un tema ampliamente abordado y aplicado en las empresas públicas y privadas, grandes - pequeñas principalmente en organizaciones nacionales e internacionales (Quispe et al., 2016). Al respecto, Leonarte(2011) indicó que, la auditoria, según el origen de los recursos auditados, se clasifica en auditoría independiente y auditoria gubernamental, esta última es el examen objetivo, pág. 18 Waynarroque Revista de Ciencias Sociales Aplicadas sistemático y profesional de las operaciones financieras $y / 0$ administrativas, efectuado con posterioridad a su ejecución, en las entidades sujetas al Sistema Nacional de Control, elaborando el correspondiente informe.

Por su parte, Alvarez (2007) indicó que, es el examen objetivo, sistemático, profesional, independiente y selectivo de evidencias de las operaciones financieras y/o administrativas efectuando con posterioridad a la gestión y al manejo de los recursos públicos con el objeto de determinar la razonabilidad financiera y presupuestal, así como evaluar el grado de cumplimiento de objetivos y metas; analizar y verificar la adquisición, internamiento, protección y empleo de los recursos físicos y financiero. En conclusión, la auditoria verifica con posterioridad operaciones financieras, administrativas y evalúa en términos de razonabilidad en base a criterios de legalidad, efectividad, eficiencia y economía.

La auditoría gubernamental tiene dentro de sus objetivos: Evaluar la correcta utilización de los recursos públicos, verificando el cumplimiento de las disposiciones legales reglamentarias; determinar la razonabilidad de la información financiera; determinar el grado en que se han alcanzado los objetivos previstos y los resultados obtenidos en relación a los recursos asignados y al cumplimiento de los planes y programas aprobados de la entidad examinada; recomendar medidas para promover mejoras en la gestión pública yfortalecer el sistema de control interno de la Entidad auditada.

Finalmente, Fonseca (2007) señaló que se distingue los siguientes tipos de auditoria: auditoría financiera, auditoria de gestión y los exámenes especiales; al respecto la Contraloría General de la República (2014) establece que los servicios de control son: servicio de control previo, simultaneo y posterior, dentro de este último se encuentran las auditorias financieras, auditorias de desempeño y las auditorias de cumplimiento.

\section{Control Gubernamental}

Chiavenatto (2007) menciona que el control es el conjunto de procedimientos, medidas y métodos coordinados que se establecen en una entidad, para salvaguardar los activos, asegurar el grado de confiabilidad de los datos contables, promover la eficiencia en el desarrollo de las operaciones y verificar el cumplimiento de las disposiciones, metas y objetivos programados; en ese entender los controles 
son medios a través de los cuales la gerencia de una entidad asegura que el sistema es efectivo y es manejado en armonía con eficiencia y economía, dentro del marco legal vigente.

Asimismo, Retamozo (2008) señaló que, el conjunto de acciones burocráticas y sociales que tiene como objetivo evaluar la eficiencia y calidad de la gestión y del gasto en el sector público. Desde esta perspectiva, el control no necesariamente se agota en el que realiza la contraloría, sino que trasciende a ella.

La ley N. ${ }^{\circ} 27785$, señala: "El control gubernamental consiste en la supervisión, vigilancia y verificación de los actos y resultados de la gestión pública, en atención al grado de eficiencia, eficacia, transparencia y económica en el uso y destino de los recursos y bienes del Estado, así como del cumplimiento de las normas legales y de los lineamientos de política y planes de acción, evaluando los sistemas de administración, gerencia y control, con fines de su mejoramiento a través de la adopción de acciones preventivas y correctivas pertinentes".

Dentro del Control Gubernamental existe el control interno que está regulado por la ley N² 28716 del 17 de abril de 2006 - Ley de Control Interno para las entidades del Estado y se encargó a la Contraloría General de la República la emisión de la normas de control interno (Congreso de la República del Perú, 2006).

Asimismo, mediante Resolución de Contraloría No 458-2008-CG de 30 del octubre de 2008 se aprobó la "Guía para la implementación del Sistema de Control Interno de las entidades del Estado" y la puso de conocimiento a todas las organizaciones públicas a fin de que fortalezcan su Sistema de Control Interno, como parte del control gubernamental (Contraloría General de la República, 2008).

\section{Informe de auditoria}

El informe de auditoría es el resultado de trabajo del auditor. Es un documento elaborado por el auditor donde se expresa de forma estándar, general y sencilla, una opinión profesional sobre los hechos evaluados durante el desarrollo de la auditoria. Presenta una serie de características fundamentales de carácter formal: es claro, oportuno sintético y se encuentra bajo un esquema de exposición determinado por la doctrina.
Un informe de auditoría se considera una herramienta esencial para informar a los usuarios de la información financiera, sobre todo en los negocios. debido a que muchos terceros usuarios prefieren, o incluso requieren información financiera certificada de un auditor externo independiente, muchos de los auditados confían en el informe de auditoría para certificar su información con el fin de atraer a los inversores, obtener préstamos y mejorar la apariencia pública.

\section{Seguimiento de medidas correctivas}

Rojas (2013) indicó que el seguimiento de medidas correctivas es una actividad que desarrollan los Órganos conformantes del Sistema Nacional de Control con el objeto de establecer el grado de superación de las observaciones efectuadas por los auditores, así como evaluar las acciones adoptadas por la administración de la entidad para implementar las recomendaciones incluidas en el informe de auditoría.

Asimismo, los Órganos de Control Interno siendo parte del sistema Nacional de Control son los encargados de realizar la verificación y seguimiento de implementación de Recomendaciones derivadas de Informes de Acciones de Control, como parte de un efectivo y oportuno ejercicio de control gubernamental.

Según la Directiva "Verificación y Seguimiento de Implementación de Recomendaciones Derivadas de Informes de Acciones de Control" la situación de las recomendaciones planteadas en los informes de auditoría, son las siguientes: pendiente, en proceso, implementada y archivado definitivo (Contraloría General de la República, 2000).

Finalmente, cabe precisar que se tienen trabajos de investigación referidos al tema planteado, realizados a otras entidades de la región Puno, siendo las más resaltantes los trabajos de: Calsin (2016) quien evaluó la implementación de las recomendaciones del Órgano de Control Institucional en la Gestión de la Sub Gerencia de Logística de la Municipalidad Provincial de Puno; Chura (2010) con su investigación referida a la evaluación de la implementación de las recomendaciones de los informes de acciones de control de la Municipalidad Provincial de San Román, Juliaca y Flores (2006) quien analizó la implementación de las recomendaciones en el Gobierno Regional de Puno, periodo 2003 - 2005. 
El objetivo de la investigación fue: Determinar el nivel de cumplimiento en la implementación de las recomendaciones planteadas en los informes de auditoría emitidos por el Órgano de Control Institucional y su incidencia en la gestión del Gobierno Regional, periodos 2013-2014.

\section{MATERIAL Y MÉTODOS}

Método analítico - descriptivo. - Este método consiste en discutir, analizar los datos, para conocer los detalles y características de todo lo que se investiga, por tanto, fue utilizado para el análisis de la información referente a los informes de auditoría y las recomendaciones derivadas de los informes de auditoría emitidos por el Órgano de Control Institucional del Gobierno Regional Puno.

Diseño de investigación. - Para realizar la investigación se utilizó un diseño no experimental y de tipo transversal o transeccional, toda vez que los datos fueron recolectado en un solo momento.

Población y muestra. - En total fueron 79 recomendaciones de las cuales 22 corresponden a informes administrativos ejecutadas por unidades ejecutoras distintas a la sede central, por tanto, tomando en cuenta este criterio se excluyeron de nuestra población a examinar, quedando así un total de 57 recomendaciones, razón por la cual, se realizó un muestreo no probabilístico (muestreo censal), debido a que por ser una población pequeña y finita se analizó el 100\% de los elementos de la población.

Materiales:

- Sistema de Auditoria Gubernamental (ExSAGU)

- Resoluciones de la Contraloría General de la Republica.

- Plan Anual de Control.

- Fichas de Observación.

Normativa relacionada al Sistema Nacional de Control.

- Cuadros estadísticos para la tabulación de datos.

\section{RESULTADOS Y DISCUSIÓN}

El análisis y evaluación a la implementación de las recomendaciones de los informes de auditoría se realizó, segregando por áreas, oficinas regionales o gerencias y subgerencias del Gobierno Regional Puno, a las cuales les correspondía implementar cada recomendación.
Estas áreas u oficinas regionales, gerencias regionales o subgerencias, según la evaluación realizada fueron las siguientes:

- Consejo Regional.

- Presidencia Regional.

- Gerencia General Regional.

- Gerencia Regional de Planeamiento, Presupuesto y Acondicionamiento Territorial.

- Gerencia Regional de Infraestructura.

- Oficina Regional de Administración.

- Órgano de Control Institucional.

- Secretaria Técnica Regional.

- Órgano Instructor de la CGR.

Teniendo como resultados que de 4 recomendaciones que correspondía implementar y darles cumplimiento al Consejo Regional, solo 1 que equivale al $25 \%$ fue implementada, 1 que equivale al $25 \%$ está en proceso de implementación y 2 que equivale al $50 \%$ quedan pendientes, tal y conforme se muestra en la tabla 1.

Tabla 1.

Recomendacionesmiplementadas por el Consejo Regional

\begin{tabular}{ccc}
\hline $\begin{array}{c}\text { Estado de las recomendaciones } \\
\text { emitidas }\end{array}$ & $\begin{array}{c}\text { No de } \\
\text { Recomendaciones }\end{array}$ & $\%$ \\
\hline Implementada & 1 & 25.00 \\
En proceso & 1 & 25.00 \\
Pendientes & 2 & 50.00 \\
\hline Total & 4 & 100.00
\end{tabular}

En la Presidencia Regional de 6 recomendaciones que correspondía implementar y darles cumplimiento, ninguna fue implementada, 4 que equivale al $33.33 \%$ están en proceso de implementación y 2 que equivale al $50 \%$ quedan pendientes, tal y conforme se muestra en la tabla 2.

Tabla2.

Recomendacionesmplementadas por la Presidencia Regional

\begin{tabular}{ccc}
\hline $\begin{array}{c}\text { Estado de las recomendaciones } \\
\text { emitidas }\end{array}$ & $\begin{array}{c}\text { No de } \\
\text { Recomendaciones }\end{array}$ & $\%$ \\
\hline Implementada & 0 & 0.00 \\
En proceso & 4 & 33.33 \\
Pendientes & 2 & 66.67 \\
\hline Total & $\mathbf{6}$ & $\mathbf{1 0 0 . 0 0}$ \\
\hline
\end{tabular}

En la Gerencia General Regional de 7 recomendaciones que correspondía implementar y darles cumplimiento, ninguna fue implementada, quedando el $100 \%$ de estas pendientes de implementar, tal y conforme se muestra en la tabla 3.

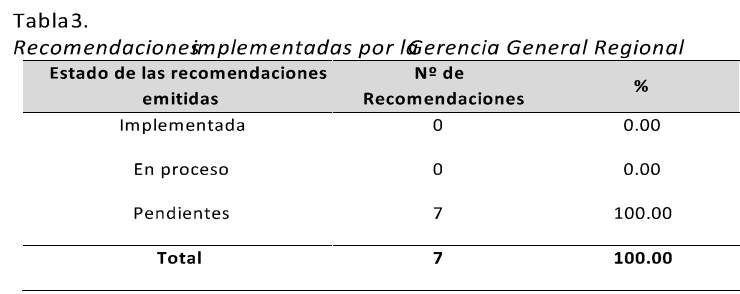


En la Gerencia Regional de Planeamiento, Presupuesto y Acondicionamiento Territorial de 5 recomendaciones que correspondía implementar y darles cumplimiento, ninguna fue implementada aun, 3 que equivale al $60 \%$ están en proceso de implementación y 2 que equivale al $40 \%$ quedan pendientes, tal y conforme se muestra en la tabla 4.

\begin{tabular}{lcc}
$\begin{array}{l}\text { Tabla4. } \\
\text { Recomendaciones implementadas }\end{array}$ & $\begin{array}{c}\text { por la Gerencia } \\
\text { Planeamiento, Presupuesto y }\end{array}$ Acondicionamiento & Rerritorial \\
\hline $\begin{array}{c}\text { Estado de las recomendaciones } \\
\text { emitidas }\end{array}$ & $\begin{array}{c}\text { No de } \\
\text { Recomendaciones }\end{array}$ & $\%$ \\
\hline $\begin{array}{c}\text { Implementada } \\
\text { En proceso }\end{array}$ & 0 & 0.00 \\
Pendientes & 3 & 60.00 \\
\hline Total & 2 & 40.00 \\
\hline
\end{tabular}

En la Gerencia Regional de Infraestructura de 7 recomendaciones que correspondía implementar y darles cumplimiento, ninguna fue implementada, que dando el $100 \%$ de estas pendientes de implementar, tal y conforme se muestra en la tabla 5.

\begin{tabular}{|c|c|c|}
\hline $\begin{array}{l}\text { Recomendacionesimplementa } \\
\text { Infraestructura }\end{array}$ & por la & Gerencia \\
\hline $\begin{array}{c}\text { Estado de las recomendacione } \\
\text { emitidas }\end{array}$ & $\begin{array}{c}\text { Node } \\
\text { Recomendaciones }\end{array}$ & s \\
\hline Implementada & 0 & 0.00 \\
\hline En proceso & 0 & 0.00 \\
\hline Pendientes & 7 & 100.00 \\
\hline Total & 7 & 100.00 \\
\hline
\end{tabular}

En la Oficina Regional de Administración de 13 recomendaciones que correspondía implementar y darles cumplimiento, ninguna fue implementada aun, 5 que equivale al $38.46 \%$ están en proceso de implementación y 8 que equivale al $61.54 \%$ quedan pendientes, tal y conforme se muestra en la tabla 6 .

Tabla6.

Recomendaciones implementadas por la Oficina Regional Administración

\begin{tabular}{ccc}
\hline $\begin{array}{c}\text { Estado de las recomendaciones } \\
\text { emitidas }\end{array}$ & $\begin{array}{c}\text { No de } \\
\text { Recomendaciones }\end{array}$ & $\%$ \\
\hline Implementada & 0 & 0.00 \\
En proceso & 5 & 38.46 \\
Pendientes & 8 & 61.54 \\
\hline Total & $\mathbf{1 3}$ & $\mathbf{1 0 0 . 0 0}$
\end{tabular}

En el Órgano de Control Institucional de 1 recomendación que correspondía implementar y dar cumplimiento, esta no fue implementada, que, dando pendiente de implementar, tal y conforme se muestra en la tabla 7.

Tabla 7.

\begin{tabular}{ccc}
\begin{tabular}{c} 
Recomendacionesmplementadas por el Órgano deontrol Institucional \\
\hline $\begin{array}{c}\text { Estado de las recomendaciones } \\
\text { emitidas }\end{array}$
\end{tabular} & $\begin{array}{c}\text { No de } \\
\text { Recomendaciones }\end{array}$ & $\%$ \\
\hline Implementada & 0 & 0.00 \\
En proceso & 0 & 0.00 \\
Pendientes & 1 & 100.00 \\
\hline Total & $\mathbf{1}$ & $\mathbf{1 0 0 . 0 0}$ \\
\hline
\end{tabular}

En la Secretaría Técnica Regional de 4 recomendaciones que correspondía implementar y darles cumplimiento, ninguna fue implementada aun, 2 que equivale al $50 \%$ están en proceso de implementación y 2 que equivale al $50 \%$ quedan pendientes, tal y conforme se muestra en la tabla 8.

Tabla 8.

\begin{tabular}{ccc}
\begin{tabular}{c} 
Recomendacionesmplementadas por la Secretaria Técnica \\
\hline $\begin{array}{c}\text { Estado de las recomendaciones } \\
\text { emitidas }\end{array}$
\end{tabular} & $\begin{array}{c}\text { No de } \\
\text { Recomendaciones }\end{array}$ & $\%$ \\
\hline Implementada & 0 & 0.00 \\
En proceso & 2 & 50.00 \\
Pendientes & 2 & 50.00 \\
\hline Total & 4 & 100.00
\end{tabular}

En el Órgano Instructor de la Contraloría General de la República de 10 recomendaciones que correspondía implementar y darles cumplimiento, ninguna fue implementada aun, 2 que equivale al 20\% están en proceso de implementación y 8 que equivale al $80 \%$ quedan pendientes, tal y conforme se muestra en la tabla 9.

\begin{tabular}{lcc}
$\begin{array}{l}\text { Tabla9. } \\
\begin{array}{l}\text { Recomendaciones implementadas } \\
\text { Contraloría General de la República }\end{array}\end{array}$ el & Órgano & Instructor de \\
\hline $\begin{array}{c}\text { Estado de las recomendaciones } \\
\text { emitidas }\end{array}$ & $\begin{array}{c}\text { No de } \\
\text { Recomendaciones }\end{array}$ & $\%$ \\
\hline Implementada & 0 & 0.00 \\
En proceso & 2 & 20.00 \\
Pendientes & 8 & 80.00 \\
\hline Total & 10 & 100.00 \\
\hline
\end{tabular}

Ahora, de acuerdo al análisis y evaluación realizada a la implementación de las recomendaciones planteadas en los informes de auditoría, y que debieron ser implementadas por las diferentes áreas, oficinas regionales, gerencias regionales y subgerencias de la entidad, se tiene el siguiente resumen:

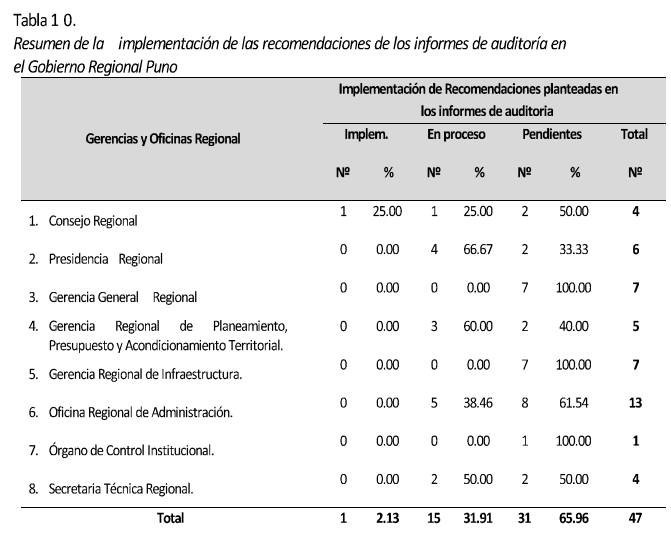

De la tabla 10, se aprecia que de las 47 recomendaciones establecidas en los informes de auditoría y que debieron ser implementadas por la gestión del Gobierno Regional Puno, solo 1 que equivale al $2.13 \%$ fue implementada por el Gobierno Regional, 15 que equivale al $31.91 \%$ se encuentran en 
proceso de implementación y 31 que equivale al $65.96 \%$ aún no se implementan y quedan pendientes. Con lo que se observa en la gestión del Gobierno Regional Puno durante el periodo: 2013 y 2014, existe un incumplimiento de la implementación de las recomendaciones, resultados que se reflejan también en la tabla 11 y figura 1 , que se muestran a continuación.

Tabla 11.

Recomendacionesmplementadas por la Gestión del Gobierno Regional Pun

\begin{tabular}{ccc}
$\begin{array}{c}\text { Estado de las recomendaciones } \\
\text { emitidas }\end{array}$ & $\begin{array}{c}\text { № de } \\
\text { Recomendaciones }\end{array}$ & $\%$ \\
\hline Implementada & 1 & 2.13 \\
En proceso & 15 & 31.91 \\
Pendientes & 31 & 65.96 \\
\hline Total & $\mathbf{4 7}$ & $\mathbf{1 0 0 . 0 0}$ \\
\hline
\end{tabular}

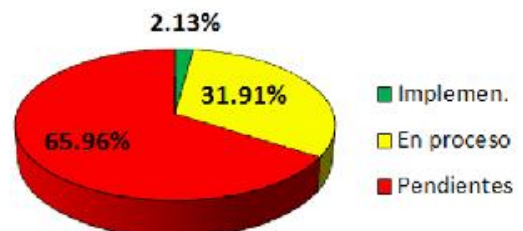

Figura 1. Implementación de las recomendaciones en el Gobierno Regional Puno

\section{Discusión}

Según Calsin (2016) en su investigación "La implementación de las recomendaciones del Órgano de Control Institucional en la Gestión de la Sub Gerencia de Logística de la Municipalidad provincial de Puno" concluyó lo siguiente: La implementación de recomendaciones emitidas por $\mathrm{OCl}$, se encuentra aún en "proceso de implementación" a pesar de que se muestre en documentos que estos ya han sido superados, pues de esto se tiene que las recomendaciones no son valoradas y tomadas con la seriedad del caso. En tal sentido, comparando los resultados obtenidos, respecto a las recomendaciones implementadas por la Oficina Regional de Administración del Gobierno Regional Puno; donde se encuentra la Oficina de Abastecimiento y Servicios Auxiliares; se tuvo 13 recomendaciones a implementar, de las cuales 5 se encuentra "en proceso" y las 8 restantes como "pendientes"; obteniendo de esta manera una similitud con las conclusiones brindadas en la investigación mencionada.

Chura (2010) en su investigación "Evaluación de la implementación de las Recomendaciones de los informes de Acciones de Control en la Municipalidad Provincial de San Román Juliaca", dentro de sus principales conclusiones destacó que esta entidad, no efectúa una adecuada implementación de las recomendaciones, al existir recomendaciones pendientes y en proceso de implementación de ejercicios anteriores, como se evidencia en los informes semestrales de Seguimiento de Medidas Correctivas, no cumpliéndose con las disposiciones emitidas al respecto por el titular, conforme lo establece la Directiva $N^{\circ}$ 14-2000-CG. Ahora comparando con los resultados obtenidos en el trabajo de investigación, se refleja la inadecuada implementación de las recomendaciones que existe también el Gobierno Regional Puno, toda vez que el $65.96 \%$ de las recomendaciones se encuentran "pendientes" de implementar, el 31.91\% "en proceso" y $\tan$ solo el $2.13 \%$ que equivale a 1 esta "implementada"; por tanto, los resultados dan una conclusión similar al estudio realizado en la Municipalidad Provincial San Román.

Flores (2006) en su investigación titulada "Análisis a la implementación de las Recomendaciones de Informes de Acciones de Control en el Gobierno Regional de Puno", concluyó que el Gobierno Regional Puno, no efectúa una adecuada implementación de las recomendaciones, al existir recomendaciones pendientes y en proceso de implementación de ejercicios anteriores, como se evidencia en los informes semestrales de seguimiento de medidas correctivas, correspondientes al periodo II semestre 2003 y 2005, no cumpliéndose con las disposiciones emitidas al respecto por el titular de la entidad, conforme lo establece la Directiva N. 014 2000-CG/B150. Asimismo, indicó que la causa fundamental por la que no existe una adecuada implementación de recomendaciones, es la falta de diligencia en el cumplimiento de sus funciones, y la no aplicación de las normas legales referente a las implementaciones de recomendaciones por parte de los responsables de la implementación; por tanto, si ellos cumplieran estrictamente sus funciones y si se preocupan por conocer los dispositivos legales referentes a la implementación de las recomendaciones, las mismas serian superadas en el tiempo menor posible, y no existirían recomendaciones pendientes o en proceso de implementación de años anteriores.

Considerando las conclusiones brindadas, se corrobora que el bajo nivel de cumplimiento en la implementación de las recomendaciones sigue manteniéndose en el Gobierno Regional Puno, toda vez que los resultados arrojados en el trabajo de investigación indican que la implementación de las 
recomendaciones en el periodo 2013 y 2014 se encuentran en estado: "en proceso" y "pendientes".

\section{CONCLUSIONES}

La falta de cumplimiento en la implementación de las recomendaciones establecidas en los informe de auditoría emitidas por el Órgano de Control Institucional del Gobierno Regional Puno, tal y conforme se demuestra en los resultados de la investigación, donde tan solo el Consejo Regional de la entidad implementó 1 de las 4 recomendaciones que le correspondía implementar; y las demás Gerencias y Oficinas Regionales, hasta el momento no implementan las recomendaciones que les corresponde; trae consigo que estas sean reiterativas y no aporten en una mejora en la gestión del Gobierno Regional Puno. Es así que realizando la evaluación y análisis de manera conjunta al cumplimiento en la implementación de las recomendaciones establecidas en los informe de auditoría, la gestión en general del Gobierno Regional Puno según los resultados dela investigación, no realizó un cumplimiento adecuado y de manera eficiente de dichas recomendaciones, toda vez que tan solo el $2.13 \%$ que equivale a una recomendación fue "implementada"; el $31.91 \%$ de las recomendaciones equivalentes a 15 de estas se encuentran "en proceso" de implementación y el $65.96 \%$ equivalentes a 31 recomendaciones están en estado "pendiente". Por tanto, se concluye que existe una deficiente implementación de las recomendaciones que dan como producto que se tenga una incidencia negativa respecto a la gestión del Gobierno Regional Puno.

\section{REFERENCIAS BIBLIOGRÁFICAS}

Álvarez, I. (2007). Auditoria Gubernamental (3era ed.; Pacifico., Ed.). Lima, Perú

Calsin, J. (2016). La implementación de las Recomendaciones del Órgano de Control Institucional en la Gestión de la Sub Gerencia de Logística de la Municipalidad provincial de Puno. (Tesis de pregrado). Universidad Nacional del Altiplano, Puno - Perú.

Chiavenatto, I. (2007). Administración de Recursos Humanos (8ava ed.; McGraw Hiil., Ed.). https://doi.org/10.1002/jsfa.7294

Chura, O. (2010). Evaluación de la implementación de las Recomendaciones de los informes de Acciones de Control en la Municipalidad Provincial de San Román Juliaca, Periodo 2008 2009. (Tesis de pregrado). Universidad Nacional del Altiplano, Puno - Perú
Congreso de la República del Perú. (2006). Ley de Control Interno en las Entidades del Estado", aprobado mediante Ley $N^{\circ} 28716$. Recuperado de http://www.pcm.gob.pe, Perú.

Contraloría General de la República. (2000). Directiva No 014-2000-CG/B150 "Verificación y Seguimiento de Implementación de Recomendaciones

Derivadas de Informes de Acciones de Control." Recuperado de http://www.contraloria.gob.pe.

Contraloría General de la República. (2002). Ley Orgánica del Sistema Nacional de Control y de la Contraloría General de la República - Ley No 27785 y modificatorias. Recuperado de http://www.contraloria.gob.pe

Contraloría General de la República. (2008). Guía para la implementación del Sistema de Control Interno de las entidades del Estado, aprobado mediante Resolución de Contraloría No 458-2008-CG. Recuperado de http://www.contraloria.gob.pe.

Contraloría General de la República. (2014). Normas Generales de Control Gubernamental, aprobado mediante Resolución de Contraloría No 2732014-CG. Recuperado de http://www.contraloria.gob.pe., Lima, Perú.

Flores, M. (2006). Análisis a la implementación de las Recomendaciones de Informes de Acciones de Control en el Gobierno Regional de Puno Periodo 2003 - 2005. (Tesis de pregrado). Universidad Nacional del Altiplano, Puno - Perú.

Fonseca, O. (2007). Auditoría Gubernamental Moderna (1era ed.). Lima, Perú: IICO S.A.

Leonarte, V. (2011). Sistema Nacional de Control. Recuperado de:

www.cal.org.pe/pdf/diplomados/10nacional.pdf.

Quispe Fernández, G. M., Arellano Cepeda, O. E., \& Ayaviri Nina, D. (2016). Aplicación de la Auditoría en las MyPEs del Ecuador: Un estudio de la demanda. Revista de Investigaciones Altoandinas - Journal of High Andean Research, 18(4), 483-496. https://doi.org/10.18271/ria.2016.241

Retamozo, A. (2008). Contrataciones y Adquisiciones del Estado y Normas de Control (6ta ed.; Grafía Editores e Impresores, Ed.). Lima, Perú.

Rojas, H. (2013). Evaluación de las Acciones de Control de Auditoria Interna de la Municipalidad Provincial de Puno. 2011 - 2013. (Tesis de pregrado). Universidad Nacional del Altiplano, Puno - Perú.

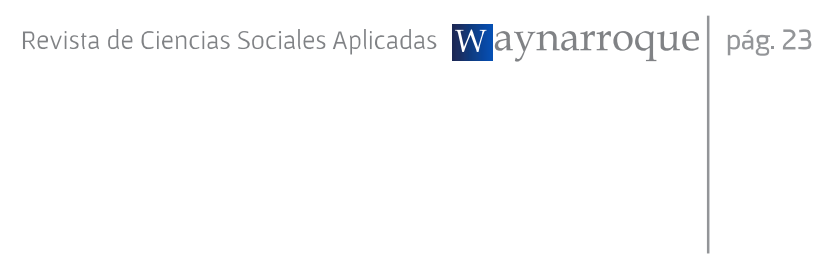

